\title{
Study of the focused laser spots generated by various polarized laser beam conditions
}

\author{
Y. H. FU*, F. H. HO*, W. C. LIN*, W.-C. LIU†\& D. P. TSAI* \\ *Department of Physics, National Taiwan University, Taipei, Taiwan 10617, R. O. C. \\ †Department of Physics, National Taiwan Normal University, Taipei, Taiwan 116, R. O. C.
}

Key words. Apodization, focal point, near-field scanning optical microscopy, polarization.

\section{Summary}

In this work, three-dimensional near-field imaging of the focused laser spot was studied theoretically and experimentally. In the theoretical simulation, we use the electromagnetic equivalent of the vectorial Kirchhoff diffraction integral to calculate the intensity distribution of the focal region, and a high depolarization is found in high numerical aperture systems (NA $=0.85$ ). The experimental set-up is based on a nearfield scanning optical microscope (NSOM) system. A high-NA objective lens is used to focus incident light of various polarizations, and a tapered near-field optical fibre probe of the NSOM system is used to determine the intensity of the focal field. The results show an asymmetric distribution of the focused intensity with the linear polarized laser beam.

\section{Introduction}

Research on the focal region is an interesting topic in both fundamental and applied optics. Important issues such as depolarization and defocusing are always taken into account in high numerical aperture (NA) systems, but they are not so obviously evident in the focusing system of lower NA. Richards \& Wolf (1959) have established a vectorial diffraction theory for systems with high NA. They found that effects due to the intrinsic vector characteristics of the electric fields are not negligible in high-NA systems, and the focus of a linearly polarized beam may be asymmetrically deformed as well. Asymmetry focus has been described in detail by Stamnes (1986). In the imaging theory of a high-NA system (Gu, 1999), effects of the apodization, depolarization and aberration were no longer a trivial issue. It is important to understand the optical field in the focal region in order to prodvide high-quality images in many applications of optical microscopy, especially

Correspondence: Dr Din Ping Tsai. Fax: +8862 23639928 ; e-mail: dptsai@phys.ntu.edu.tw in high-resolution and three-dimensional optical microscopy. A few studies of the optical field at the focal region were performed by using a charge-coupled device (CCD) camera (Gibson \& Lanni, 1991), a knife-edge method (Quabis et al., 2001) and near-field fibre probe (Rhodes et al., 1998). In this paper, we describe the investigation of the focal region of a highNA objective lens directly using a near-field scanning optical microscope (NSOM). High spatial resolution and better accuracy of the near-field measurements are expected for further understanding of the focusing properties of high-NA lenses.

\section{Theoretical simulation}

To calculate the intensity distribution of the focal region, we used the electromagnetic equivalent of the vectorial Kirchhoff diffraction integral developed by Visser \& Wiersma (1992). We assume the incident plane waves are normal to the lens that transforms plane waves into spherical waves, and focuses them to a point. The schematic is shown in Fig. 1. The incident field $E_{\text {inc }}$ is transferred to $E_{s}$ on the reference sphere through a coordinate transformation matrix. The intensity distribution of the focused fields is integrated using the vectorial KirchhoffFresnel diffraction formula. Equations (1) and (2) are KirchhoffFresnel integrals for electric and magnetic fields in the focal regions, respectively, and $\mathbf{G}$ is the Green function with suitable boundary conditions.

$$
\begin{aligned}
& \mathbf{E}(x)=\int_{s}\left[\left(-i k \mathbf{G}+\nabla \mathbf{G} \cdot \hat{n}\left(\nabla \mathbf{G} \cdot \mathbf{E}_{\mathbf{s}}\right)\right] d \sigma\right. \\
& \mathbf{B}(x)=\int_{s}\left[\left(-i k \mathbf{G}+\nabla \mathbf{G} \cdot \hat{n}\left(\nabla \mathbf{G} \cdot \mathbf{B}_{\mathbf{s}}\right)\right] d \sigma\right.
\end{aligned}
$$

where $d \sigma$ is a surface element,

$$
\begin{aligned}
\mathbf{G}(\mathbf{p}, \mathbf{x}) & =\frac{\exp (i k|\mathbf{p}-\mathbf{x}|)}{4 \pi|\mathbf{p}-\mathbf{x}|}, \\
\nabla \mathrm{G}(\mathrm{p}, \mathrm{x}) & =\left(\frac{1}{|\mathrm{p}-\mathrm{x}|}-i k\right) \mathrm{G} \hat{e}_{\mathrm{G}} \\
\hat{e}_{G} & =\frac{(\mathbf{x}-\mathbf{p})}{|\mathbf{x}-\mathbf{p}|}
\end{aligned}
$$




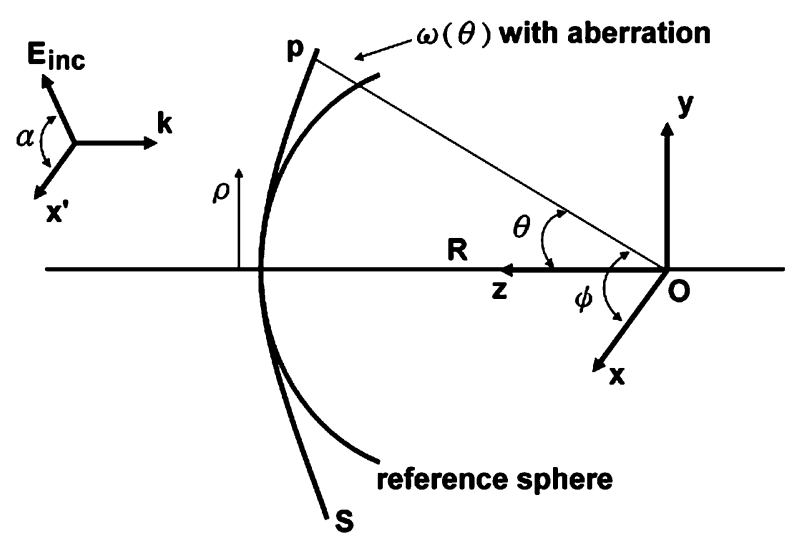

Fig. 1. Schematic of plane waves focusing through a lens onto a focal point.

The diffracted field is obtained by integrating over the wave front of the spherical wave. In our simulation, we investigate variations in the linear polarization of the electromagnetic field at the focal region for a high-NA lens. The results are shown in Fig. 2. The contour plot of the intensity distribution for a lens of $0.85 \mathrm{NA}$ is shown in Fig. 2(a). In order to verify that the differences result solely from the NA, the plot of the intensity contour maps are prepared with dimensionless units, $v$ and $u$, which are defined in Eqs (6) and (7), i.e.

$$
\begin{gathered}
v=k \sqrt{x^{2}+y^{2}} \sin \Omega \\
u=k \cdot z \cdot \sin ^{2} \Omega \quad \Omega \text { : semi-angle of aperture }
\end{gathered}
$$

$v_{x}$ and $v_{y}$ are the two orthogonal directions in the transverse plane. Polarization of the incident beam is along the axis of $v_{x}$ for the results shown in Fig. 2(a,b). A similar contour plot for an NA of 0.4 is displayed in Fig. 2(b). In comparison to the result shown in Fig. 2(b), the asymmetry and depolarization of the intensity contour plot can be seen clearly along the direction of $v_{y}$ in Fig. 2(a). Distortion from a perfect circular focusing spot appears, and small features related to the polarization emerge. Figure 2(c) represents the section curve of the intensity distribution along the optical axis of $u_{z}$. Peak $\mathrm{A}$ is the maximum intensity at the focusing point, and point $\mathrm{B}$ is at the first minimum intensity along the optical axis of $u_{z}$.

\section{Experimental set-up}

A tapping-mode tuning-fork near-field scanning optical microscope (TMTF-NSOM) is used in our three-dimensional direct measurements (Tsai \& Lu, 1998, Tsai et al., 1999) of the focusing spot. A schematic of the experimental set-up is shown in Fig. 3. The tuning fork is commercially available with an electrical resonance quality $Q$ of 80000 and a resonance frequency of $32.768 \mathrm{kHz}$. A near-field optical fibre probe pulled by a commercially available device, and subsequently coated

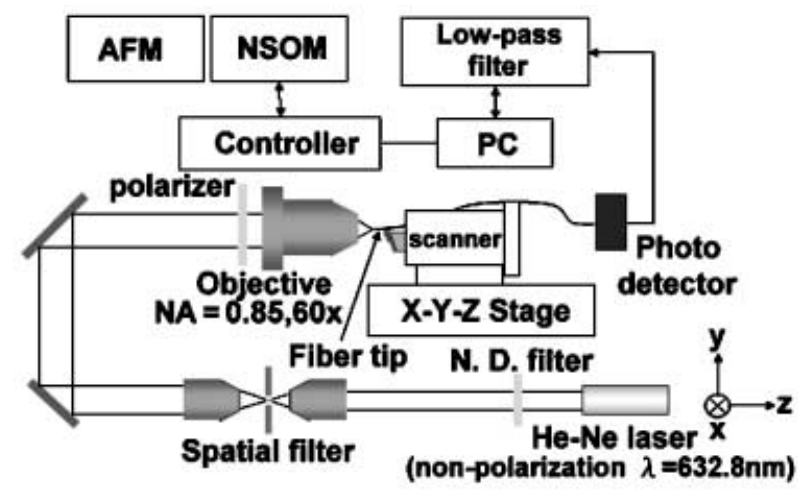

Fig. 3. Schematic diagram of the experimental set-up for measurement of the intensity distribution at the focus. (a)

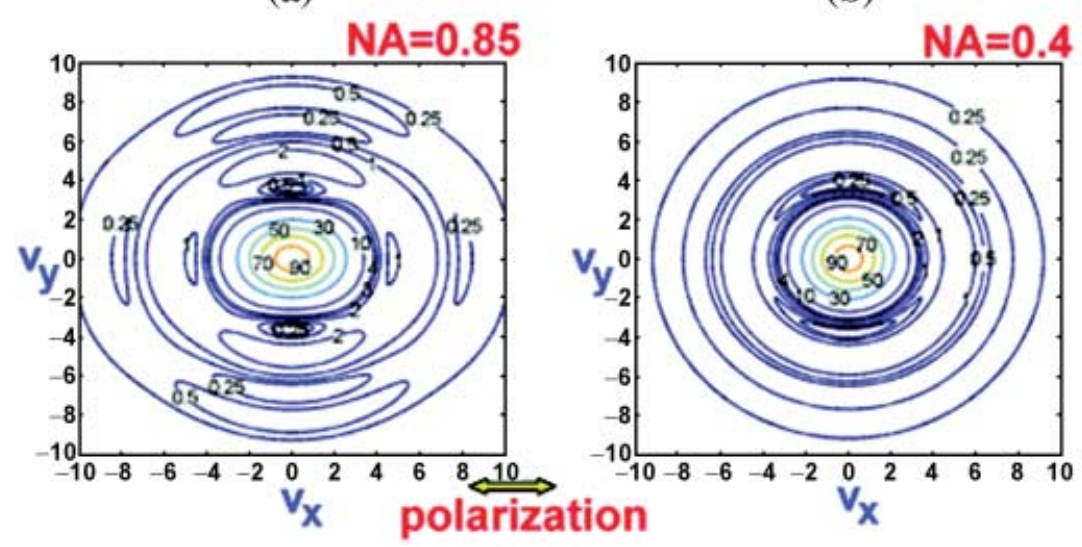

(c)

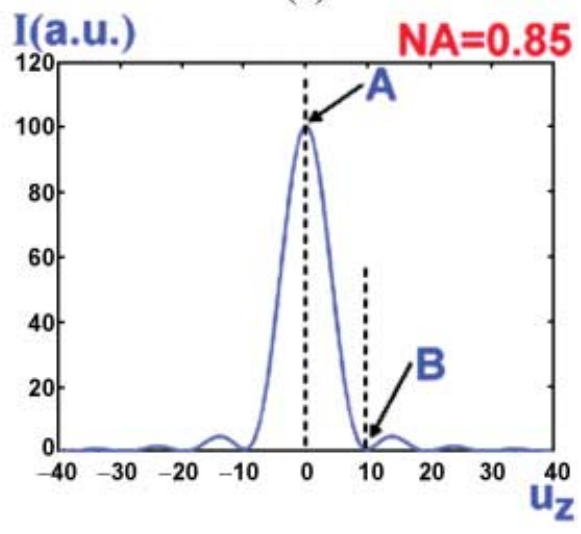

Fig. 2. Simulation results of the intensities of the polarized focusing spot with different numerical aperture (NA). The contour plots of the intensity distribution of objective lens with NA of 0.85 and 0.4 are shown in (a) and (b), respectively. (c) The section curve of the intensity distribution along the optical axis of $u_{2}$. 
with a thin layer of aluminium, is attached by epoxy at the end of one tine (the tine opposite the mechanically excited one) of the tuning fork. Optical radiation coupled to the tapered fibre tip operated in the near field was detected by a photo-diode or photomultiplier tube. Optical intensity at the focusing region is determined with high spatial resolution in the collection mode. The feedback of the tapping-mode tuning fork is shut off during our experiments.

To measure the intensity distribution of the focused laser spot for various input fields experimentally, the desired input-field distribution was focused by a microscope objective. A random polarized continuous wave $(\mathrm{CW})$ helium-neon $(\mathrm{HeNe})$ laser with a wavelength of $632.8 \mathrm{~nm}$ is used as the light source. An adjustable polarizer controls the polarization of the incident light beam. The measured spatial resolution depends mainly on the aperture of the near-field optical fibre tip, normally less than $100 \mathrm{~nm}$. The $z$-axis shown in Fig. 3 is the optical axis for focusing, and the $x-y$ planes are transverse planes parallel to the focal plane.

\section{Results and discussion}

For a focusing objective with NA of 0.85 , the measured optical intensity distribution at the focal region is shown in Figs 4 and 5. Figure 4 displays the intensity distributions of different polarization of the incident beam measured at the focal plane. The focal plane here is at point A shown on Fig. 2(c). Figure $4(a, b, c)$ show the intensity distribution measured with random polarized light, and polarized along the $x$-axis, and $y$-axis, respectively. Figure 4(d) shows the calculated result of the simulation image at the focal plane with polarization along the $x$-axis. Two rows of the section profiles are shown in Figure 4(a-d). These are the section curves along the $x$-axis and $y$-axis for each image, respectively. Results show that the focused image of the random polarized light beam agrees well with the calculation, but the elongation of the focused axis in Fig. $4(\mathrm{~b}, \mathrm{c})$ is opposite to the results of simulation calculated in Fig. 4(d). The measured ratios of the full-width-half-maximum (FWHM) of the short axis and long-axis of Fig. 4(b,c,d) are 0.803, 0.785 and 0.789, respectively. Both 0.803 and 0.785 are in a very reasonable agreement with the calculated value of 0.789 . These interesting results may come from fibre probe artefacts or the aberration from focusing objective lens. We tried different fibre probes and obtained repeatable results. To further verify the hypothesis, we scanned the plane of the first minimum along the optical axis, i.e. point B shown in Fig. 2(c). Figure 5(a,b,c) are the imaging results at the first minimum point. Figure 5(a) is the image using a random polarization light source, and it agrees well with the calculated results shown in Fig. 4(a). Figure 5(b,c) are the images of the measurements with the polarization along the $x$-axis and the $y$-axis, respectively. It is not surprising that the results agree well with the calculated simulation. Figure 5(d) is the image of the simulation result with the polarization along the $x$-axis at the first minimum focusing point. Based on the results of the measured images from both the maximum at
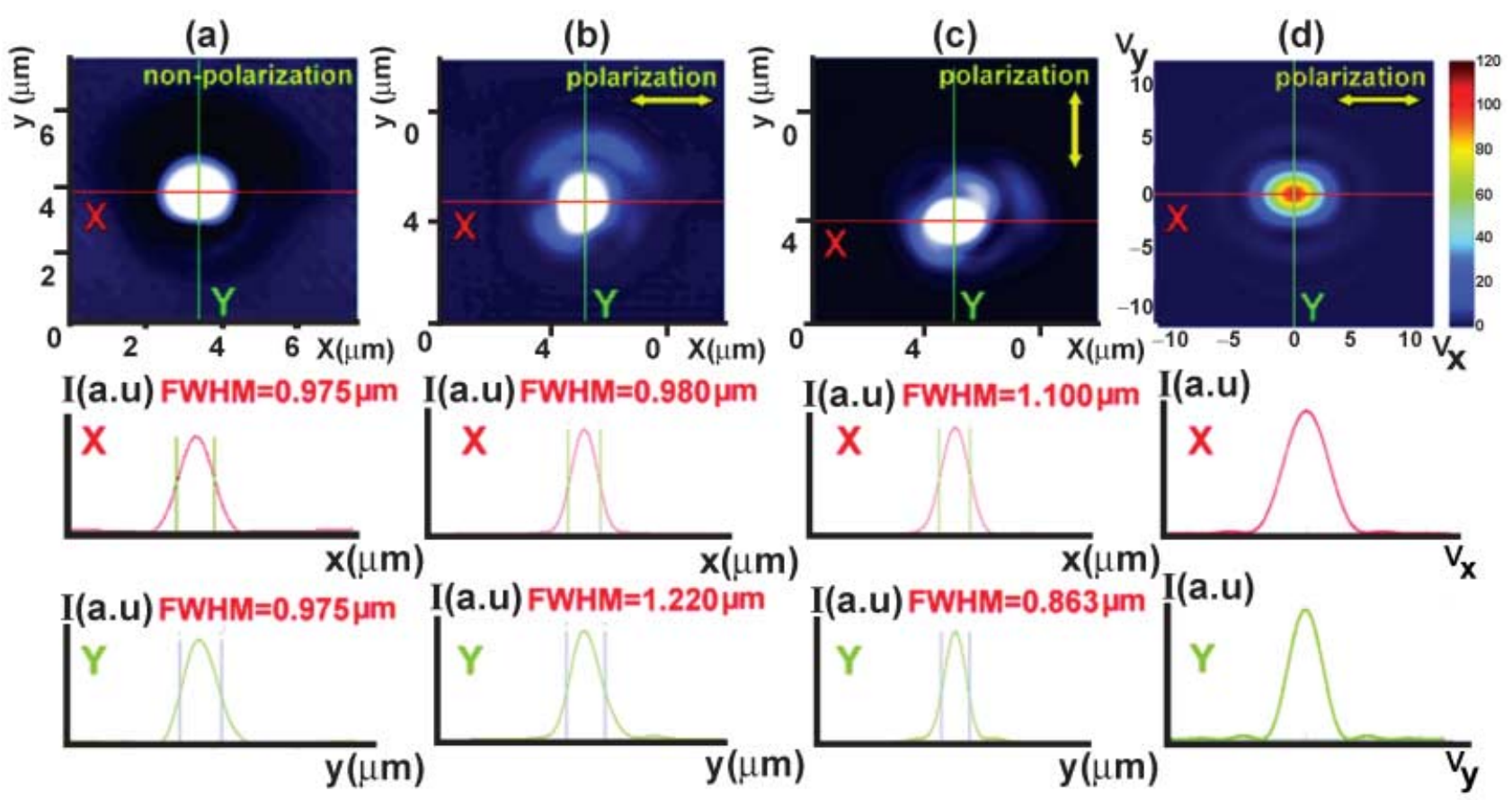

Fig. 4. Images measured at the maximum of the focusing laser spot. (a) Image and section curve of the random polarized light source. (b,c) Images and section curves of a linear polarized light source along the $x$-axis and the $y$-axis, respectively. (d) Image and section curve of calculated simulation of a linear polarized light source along the $x$-axis. 
(a)
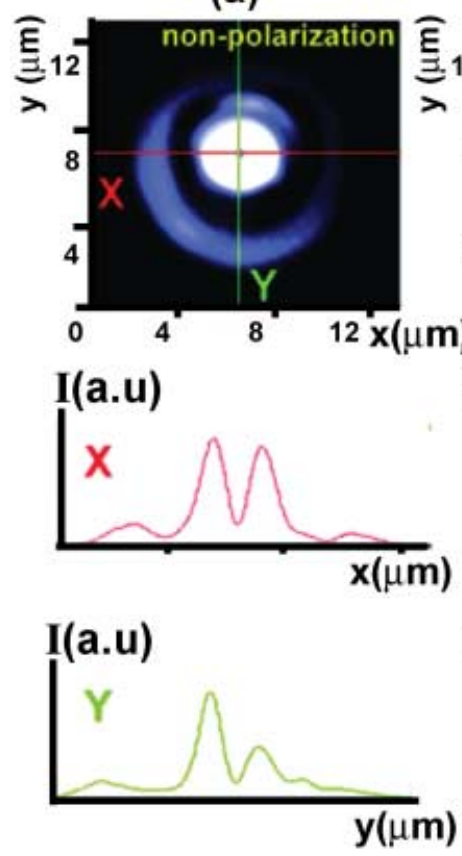

(b)

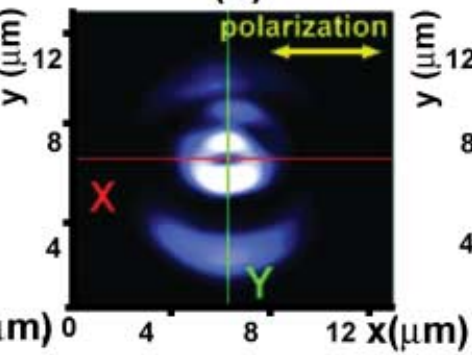

I(a.u)

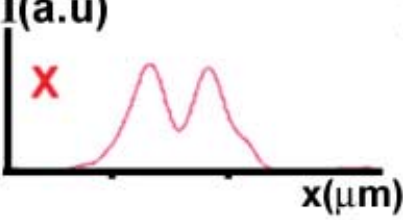

I(a.u)

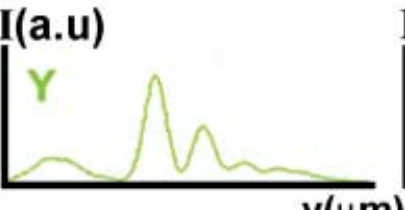

(c)
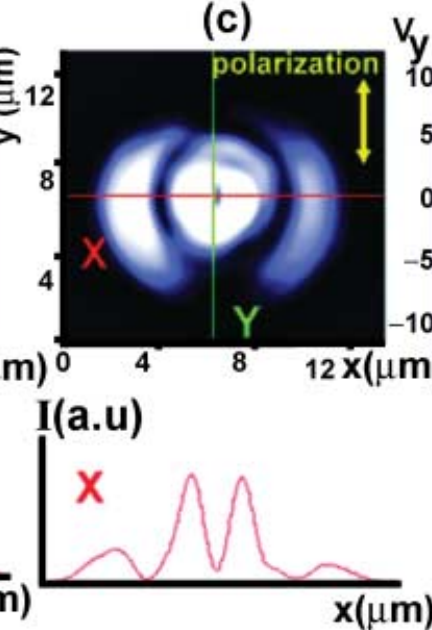

I(a.u)

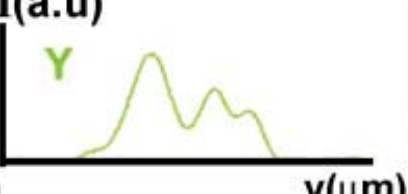

(d)
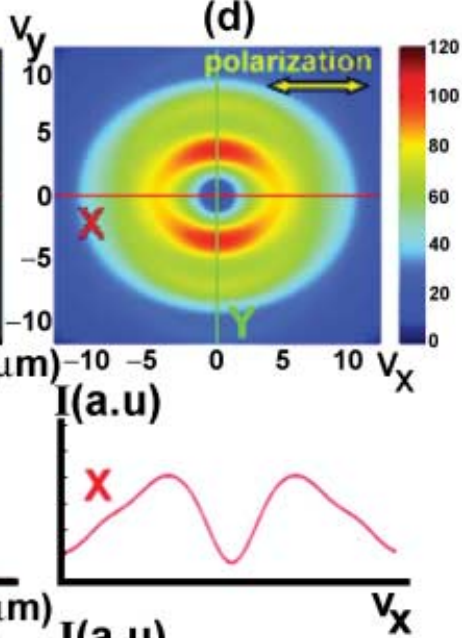

Fig. 5. Images measured at the first minimum along the optical axis. (a) Image of the random polarized light source. (b,c) Images of a linear polarized light source along the $x$-axis and the $y$-axis, respectively. (d) Image of calculated simulation of a linear polarized light source along the $x$-axis.

the focus and the first minimum along the optical axis, we believe that the aberration of the focusing objective lens is the main cause of the results of Fig. 4(b,c).

\section{Conclusions}

We have directly measured the intensity distribution at the focal region using a TMTF-NSOM. The diffraction pattern at the focus spot, the size of the focusing spot, symmetry of the focusing spot of a random polarized light source, symmetry of the focusing ring at the first minimum, and elongation or polarization change of the focusing objective were observed. The intensity distribution of the first-order diffraction pattern agrees well with the results of simulation. Interesting aberration effects of the focusing lens were found and studied. The results show that polarization and aberration play an important role in the focusing of a high-NA lens.

\section{Acknowledgements}

We gratefully acknowledge the research support by the National Science Council of R.O.C. for financial support under Grant Nos. NSC-89-2112-M-002-083, NSC-89-2622L-002-001, NSC-90-2622-L-002-003 and NSC90-2112-M-
002-047. This study was also supported by MOEA under Grant No. 91-EC-17-A-08-SI-0006.

\section{References}

Gibson, S.F. \& Lanni, F. (1991) Experimental test of an analytical model of aberration in an oil-immersion lens used in three-dimensional light microscopy. J. Opt. Soc. Am. A, 8, 1601-1613.

Gu, M. (1999) Advanced Optical Imaging Theory. Springer, Berlin.

Quabis, S., Dorn, R., Eberler, M., Glöckl, O. \& Leuchs, G. (2001) The focus of light-theoretical calculation and experimental tomographic reconstruction. Appl. Phys. B, 72, 109-113.

Rhodes, S.K., Barty, A., Roberts, A. \& Nugent, K.A. (1998) Subwavelength characterization of optical focal structures. Optics Commun. 145, 9-14.

Richards, B. \& Wolf, E. (1959) Electromagnetic diffraction in optical system II. Structure of the image field in an aplanatic system. Proc. $R$. Soc. London, Series A, 253, 358-379.

Stamnes, J. (1986) Waves in Focal Regions. A. Hilger, Bristol.

Tsai, D.P. \& Lu, Y.Y. (1998) Tapping-mode tuning fork force sensing for near-field scanning optical microscopy. Appl. Phys. Lett. 73, 2724-2726.

Tsai, D.P., Yang, C.W., Lo, S. \& Jackson, H.E. (1999) Imaging local index variations in an optical waveguide using a tapping-mode near-field scanning optical microscope. Appl. Phys. Lett. 75, 1039-1041.

Visser, T.D. \& Wiersma, S.H. (1992) Diffraction of converging electromagnetic waves. J. Opt. Soc. Am. A, 9, 2034-2047. 